\title{
STUDY OF COMPOSITE INSULATOR SHEDS SUBJECTED TO WHEEL TEST
}

\begin{abstract}
The paper presents investigation of the properties of the surface and the material stiffness - flexibility of series of samples taken from the sheds of the composite insulators. The insulators were previously subjected to wheel test. The wheel test and $1000 \mathrm{~h}$ salt fog test are regarded as alternative examination of the material resistance to the effects of electrical surface discharges. There were investigated two series of the samples of the composite insulators sheds. Examined specimens, made of HTV silicone rubber, were taken from the sheds of medium-voltage composite insulators of two different manufacturers. Insulators of both types passed the $1000 \mathrm{~h}$ salt fog test without reservation. Meanwhile, the wheel test can provide a basis for better distinguishing between physical properties of the tested materials. In the case of the insulators of one of the manufacturers the wheel test result was negative. Cross puncture effect of the sheds took place in several places. In addition, sheds were covered with dark coating of varying thicknesses. The results of the study indicated a significantly stronger influence of electrical and temperature factors on the sheds under investigations during the wheel test than in the case of the $1000 \mathrm{~h}$ salt fog test. It can be stated that these tests cannot be considered as alternative and it seems that wheel test enables better distinguishing between properties of the materials.
\end{abstract}

Keywords: composite insulators, silicone rubber, wheel test, electrical surface discharges, tracking and erosion

\section{Introduction}

The wheel test [1] known also in literature as rotating wheel dip test (RWDT) [2] is a method of testing of composite insulator housing materials for tracking and erosion. An alternative method of testing the housing materials resistance to electrical surface discharges is the $1000 \mathrm{~h}$ salt fog test described in the IEC standard [3] and call further in this paper a salt fog test. In this revised IEC standard the salt fog test is described and standardized, while the wheel test has been withdrawn from the standard, along with the $5000 \mathrm{~h}$ test at multiple stresses.

Despite this fact, the experience of the Institute of Power Engineering shows that the wheel test and salt fog test can hardly be considered as alternative tests [4]. It seems that application of the wheel test gives better assessment of the resistance of-housing material of composite insulator sheds to electrical surface discharges than application of the salt fog test.

Bearing the above in mind, the decision was made to perform comparative tests by means of the wheel test method of two types of composite insulators of a similar chemical composition of housing material. Simultaneously, for the evaluation of the results of these comparative examinations, with agreement to the requirements of the documents [1,3], surface tomography and force-penetration depth characteristics examinations were adopted in addition.
The study presents the results of testing - surface parameters and stiffness-vulnerability of a batch of samples of composite insulator sheds, which were previously subjected to the wheel test. The test comprised two series of the samples of medium voltage (MV) composite insulators sheds of various manufacturers - designated as $P f$ and Lt. All samples were cut out of the sheds and were made of HTV (high temperature vulcanised) silicone rubber, at present called also HCR (high consistency rubber).

The insulators of both types had passed, beyond doubts, the salt fog test. Yet, in the case of the wheel test, distinct changes occurred in the condition of the surface of the sheds. The insulator sheds became covered with a dark coating of different thickness and sometimes cracks - particularly in the case of $P f$ samples were observed. There were also light traces left by the surface discharges. First of all, however, in the case of $P f$ sheds, cross punctures occurred in several places. As a consequence, the result of the wheel test for these insulators was found negative.

The work presents the results of surface topography testing (profile images and their parameters) as well as of an examination of the force-penetration depth characteristics (P-h) by means of the microindentation method. The results of the tests enabled assessment of the degree of defecting of the silicone rubber surface due to the operation of electric surface discharges during the wheel test. On this basis, it was possible to compare both

\footnotetext{
* INSTITUTE OF POWER ENGINEERING, 8 MORY STR., 01-330 WARSZAWA, POLAND

** INSTITUTE OF FUNDAMENTAL TECHNOLOGICAL RESEARCH POLISH ACADEMY OF SCIENCES, 5B PAWIŃSKIEGO STR., 02-106 WARSZAWA, POLAND

\# Corresponding author: pranach@ippt.pan.p
} 
materials under examination in terms of resistance to tracking and erosion. In addition, conclusions were drawn regarding the compatibility of the salt fog test and the wheel test.

\section{Objects under investigation and the measurement procedure}

Tests of the original material properties were performed on two groups of samples taken from medium voltage composite insulators. The first one comprised elements of the top, middle and bottom insulator shed, designated as $P f$. The second sample group consisted of analogous fragments of another manufacturer's insulator $-L t$. Both insulators were factory new and subjected to no other prior testing. The samples, presented in Figure 1, were used for test surface topography - profile images and their parameters, followed by a mechanical examination of the force-penetration depth characteristics. The surface topography testing was performed both on the upper and lower sides of all samples.
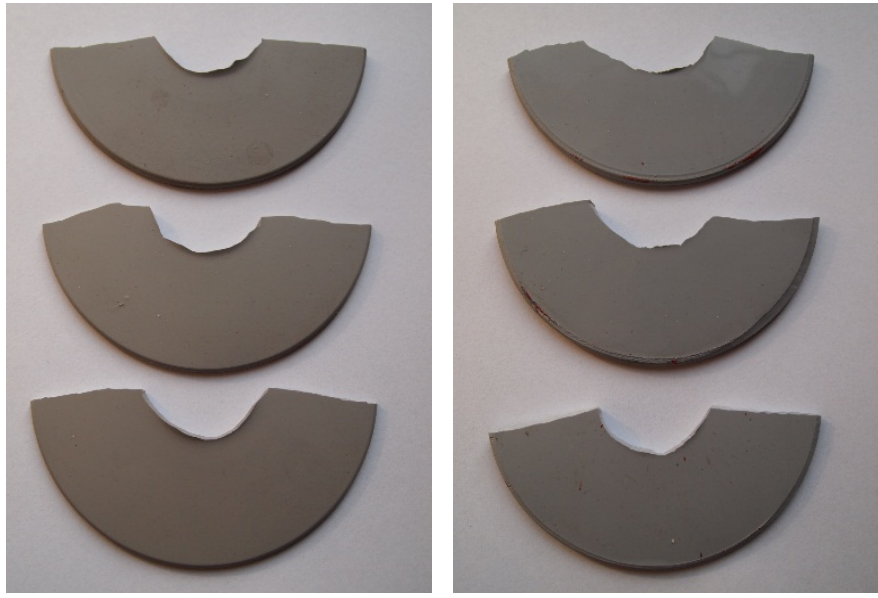

Fig. 1. The insulator shed samples designated as $P f-$ the top, middle and bottom (the upper side of the sheds) - on the left, and the samples designated as $L t$ (the bottom side of the sheds) - on the right

Both MV insulators $-P f$ and $L t-$ had similar shapes and dimensions. Chemical testing of the samples under examination was performed as well, which demonstrated a very high degree of similarity between both HTV (HCR) silicone rubbers. The amount of alumina, which facilitates the exchange of heat, equalled ca. $30 \%$. The content of silica, which poses mechanical reinforcement of the material, totalled $18 \%$, with about $50 \%$ share of the organic polymer. The density (ca. $1.5 \mathrm{~g} / \mathrm{cm}^{3}$ ), porosity (ca. $0.25 \%$ ) and thermogravimetric characteristics of both materials were nearly the same. Despite this, the surface of the $P f$ samples was matt - deprived of gloss and slightly rough to the touch. The sheds were also significantly less thick than in the case of $L t$ sheds. The surface of the $L t$ samples was glossy and smooth to the touch. The sheds were also not only considerably thicker, but also perceptibly stiffer than in the case of $P f$ ones. Hence, different manufacturing process by both insulator manufacturers had to contribute to the physical properties of the rubbers.

The insulators of both types passed the salt fog test successfully [3]. The salt fog test, was lasting 1,000 hours and was conducted for two identical insulators subjected to sprayed saline, under alternating voltage - selected to match the leakage path of the samples. The test was performed in a special chamber of an appropriate size (below $15 \mathrm{~m}^{3}$ ). The fog of proper contents of dissolved $\mathrm{NaCl}\left(1 \div 8 \mathrm{~kg} / \mathrm{m}^{3}\right)$ and sprayed at a constant flow rate (ca. $0.3 \mathrm{l} / \mathrm{m}^{3} \mathrm{~h}$ ), filled the entire chamber. The result of a test is regarded as positive if no punctures have occurred on both samples regarding the sheds and housing. No tracking traces (conductive paths) should be observed too. The depth of any erosion must not exceed $3 \mathrm{~mm}$ and reach the core.

The insulators of both types were then subjected to a wheel test [1]. Its results for samples $P f$ and $L t$ were significantly different, therefore these are presented in two separate chapters. The wheel test was also performed on two insulators of identical structure. They were mounted on a wheel and went through four positions during one cycle-Figure 2. In each of these positions, the sample remained still for about $40 \mathrm{~s}$, the rotation to reach the next position took ca. $8 \mathrm{~s}$. In the first part of the cycle, the insulator was submerged in a saline solution $(\mathrm{NaCl}$ content $1.4 \mathrm{~kg} / \mathrm{m}^{3}$ ), the excess of which was drained during the second part of the cycle. In the third part of the cycle, the sample was subjected to alternating voltage of the mains frequency. The test voltage is selected to match the leakage path of the samples. Surface discharges may then occur on the slightly wetted surface, across dry areas. This causes heating of the sample surface, which is cooled down during the fourth - the last - part of the cycle. The test duration is 30,000 cycles. The result of a wheel test is reported as positive in very similar conditions to the ones regarding the salt fog test.

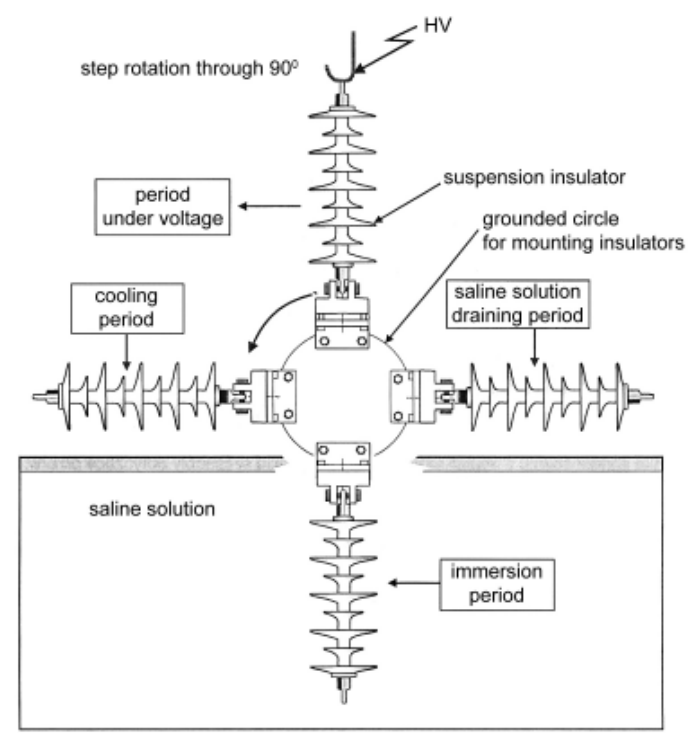

Fig. 2. The test system for rotating wheel test

Samples $P f$ and $L t$, following the wheel test, were subjected to surface topography examination. The measurements of the 
surface topography (series of subsequent profiles constituting surface image) were performed using a scanning profilometer manufactured by the German company Hommelwerke, type T8000 NANOSCAN. The measuring used the profiling method - the measurement data were collected at defined points, in perpendicular directions $\mathrm{X}$ and $\mathrm{Y}$ (bidirectional scanning) with a preset sampling grid. The minimum measuring resolution was $0.1 \mathrm{~mm}$ in the $\mathrm{X}$ direction, $0.5 \mu \mathrm{m}$ in the $\mathrm{Y}$ direction and $0.68 \mathrm{~nm}$ in the vertical direction $Z$. The profilometer was equipped with an interferometric system to measure the displacement in the $Z$ direction. The measurement data were generated considering the probe tip radius compensation $(2 \mu \mathrm{m})$ regarding the shape of the micro-asperities measured. The profile roughness parameters were determined according to the standard [5].

Surface profile examination was made along $1 \mathrm{~cm}$, taking five measurements at distances of $8 \mu \mathrm{m}$. Taking multiple measurements reduced the probability of recording random surface damages. Measurements were performed in 6 randomly chosen places, both for $P f$ and $L t$ samples. The upper and bottom surface of the top and bottom shed were examined, within areas without visible accidental scratching, pricks or other surface defects.

The examination of the force-penetration depth characteristics (P-h), performed by means of the microindentation method, was made using an instrumented microindentation tester manufactured in IFTR PAS in Warsaw [6]. The indenter tip was a ball made of tungsten carbide (WC), $1 \mathrm{~mm}$ in diameter. The force value was measured by means of a tensometric bridge, whereas displacement of the tip was measured by means of three optical sensors. The device was specifically adapted to test samples of various materials, even when the upper and bottom sample surfaces were not enough flat and parallel to each other.

\section{Examination of surface topography of $\boldsymbol{P} f$ and $L t$ samples before the wheel test}

Applying the research method, as presented above, as well as surface parameter measuring system, reference measurements were taken of $P f$ and $L t$ samples, which had not been subjected to any other testing. The following parameters, characteristic for the surface profile, were calculated [5]:

$\mathrm{Ra}$ - arithmetic mean deviation of the assessed profile;

$\mathrm{Rq}$ - Root Mean Square (RMS) deviation of the assessed profile;

$\mathrm{Rp}$ - maximum profile peak height within a sampling length;

$\mathrm{Rv}$ - maximum profile valley depth within a sampling length;

$\mathrm{Rz}$ - maximum height (interval) of the profile within a sampling length $(\mathrm{Rp}+\mathrm{Rv})$.

The results of the $P f$ and $L t$ sample surface parameter measurements were collected in Table 1. Their dispersion, taking into account the specificity of the research, can be regarded as relatively small.
TABLE 1

The surface profile parameters obtained for the $P f$ and $L t$ insulator shed reference samples; the values provided in micrometres

\begin{tabular}{|l|c|c|}
\hline \hline \multicolumn{1}{|c|}{ Parameter/Sample designation } & Pf & Lt \\
\hline Ra mean deviation & $1.1 \div 1.3$ & $0.5 \div 0.8$ \\
\hline Rq RMS deviation & $1.4 \div 1.6$ & $0.7 \div 1.0$ \\
\hline Rp max. peak height & $3.3 \div 4.1$ & $3.0 \div 3.4$ \\
\hline Rv max. valley depth & $3.4 \div 4.1$ & $2.4 \div 4.1$ \\
\hline Rz max. height Rp $+\mathrm{Rv}$ & $6.9 \div 8.2$ & $5.4 \div 7.5$ \\
\hline
\end{tabular}

The examination of the surface profile offered to conclude that the sample parameter recurrence was satisfactory. This referred to both $P f$ and $L t$ samples. The differences regarding the values of the statistical parameters ( $\mathrm{Ra}$ and $\mathrm{Rq}$ ) for both materials are not surprising, taking into consideration the dissimilarities, as visible to the naked eye, in gloss and perceptible roughness of both rubbers. The Rp, Rv parameters, and the more so their sum $\mathrm{Rz}$ - remain at a similar level in both cases. On the other hand, the statistical parameters are clearly different. The arithmetic mean deviation of surface profile amplitudes - $\mathrm{Ra}-$ is about twice as high for the $P f$ samples as for the $L t$ samples and exceeds $1 \mu \mathrm{m}$. The value of the Rq parameter - the effective value of surface profile amplitudes (RMS) - is higher, too. Irrespective of the values of the parameters collected in Table 1, a much bigger density of amplitudes was demonstrated on the $L t$ sample profiles.

\section{Examination of surface topography of $P f$ samples after the wheel test}

On the surface of all $P f$ samples, following the wheel test, the presence of a dark coating was reported. Its thickness and distribution varied for individual samples. It was also reported that in the case of both materials under examination ( $P f$ and $L t$ ), the dark layer of the coating on the surface of the sheds could easily be removed (wiped off), uncovering the surface - which parameters were typical for the original samples. Still, in the case of the $P f$ samples, a strong effect of the dark layer of contamination on the surface profile parameters was demonstrated. Wherever the layer of the coating was more distinct, changes in the surface profile image were recorded. A significant increase of the unevenness amplitude occurred, to be clearly visible on surface profile graphs (Figs. 6 and 7). The registered surface parameters increased (Table 2 and 3), and their increase was in proportion to the thickness of the layer. The parameters increased twice to more than four times, in relation to the reference value (sample surface not defected thermoelectrically). The layer thickness, as measured at the strong blackening of the surface, amounted from less than $20 \mu \mathrm{m}$ to over $30 \mu \mathrm{m}$. The surface unevenness became smaller as the blackening intensity decreased, i.e. along with a decrease in the thickness of the layer of the coating. Some samples also demonstrated the presence of light paths of erosive nature. These constituted valleys of several up to ca. $20 \mu \mathrm{m}$. The valleys were clearly reflected in surface profile images and resulted in an increase of the surface parameters. 
Using the EDS (energy dispersive spectroscopy) technique, an analysis was made of the chemical composition of the dark coating on the $P f$ samples following the circular test - Figure 3. As assumed, the examination demonstrated the presence of coal, silicon, oxygen and aluminium - the components of the HTV silicone rubber, which was filled with alumina and silica. The other elements came from the solution and the wheel test system.

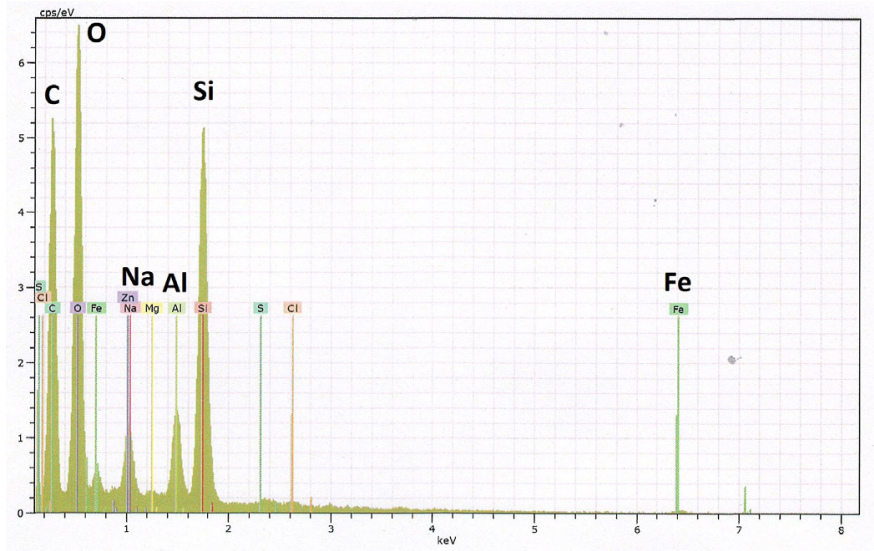

Fig. 3. The EDS diffractogram of the dark coating on the $P f$ sample surface after the wheel test

The most critical damage which was observed on two out of six $P f$ samples under examination, was cross punctures of the shed. As already mentioned, a puncture of the shed as a result of electric discharges means that the result of the test - both the wheel and the salt fog ones - is reported as negative.

The examination of the surface parameters of the insulator sheds provided by the manufacturer designated as $P f$ after the circular test, was made on 6 shed samples. The samples were taken from top and bottom sheds, as well as ones located in the middle of the height of two insulators. Both upper and lower surfaces were tested. As exemplary and representative, resulting from a great number of tests made on both surfaces of the 6 Pf samples after the wheel test, the test was presented of the middle shed sample of one of the two insulators under examination. The middle shed sample demonstrated clearly marked effects of electric discharges, a visible cross puncture in particular. Figure 4 shows the upper and Figure 5 - the lower surface of the sample, having marked the areas under examination. The area of the puncture, containing a crater, is - for obvious reasons characterized by strongly changed surface parameters. Therefore, presentation of the results of the measurements regarding this area was abandoned.

On the upper side of the sample, the area of the shed neighbouring the cut-off insulator core was examined. It was covered with a dark layer of coating the thickness of which was decreasing towards the edge of the shed. The measurement was being taken in this direction, which was clearly reflected on the registered surface profile-Figure 6 . The profile parameters were presented in Table 2. These are raised in relation to the original surface (Table 1), however, to a lesser extent than it was observed for the surfaces of strong covering with a layer of black sedi-

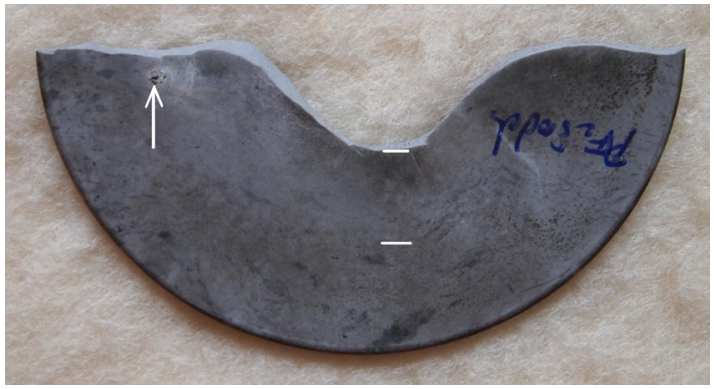

Fig. 4. The upper surface of the $P f$ insulator middle shed sample. The arrow points at the place where the shed was punctured. The lines denote the area in the vicinity of the cut-off insulator core in which the surface profile was examined. The measurement was taken downwards - towards the shed edge

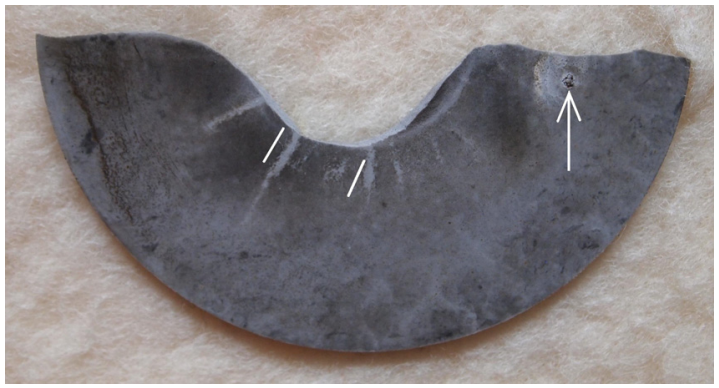

Fig. 5. The lower surface of the $P f$ insulator middle shed sample. The arrow points at the place where the shed was punctured. The lines denote the area in which the surface profile was examined. The measurement was taken from the left to the right hand side

ment. This resulted from a significant decrease in the thickness of the dark layer - the further from the place where the insulator core was cut off, the more. Hence, Figure 6 clearly shows a gradual decrease of the amplitude of the registered surface unevenness.

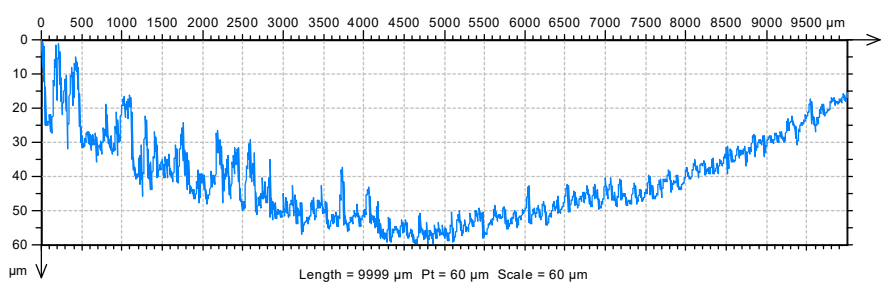

Fig. 6. The upper surface of the $P f$ insulator middle shed sample - the image of the surface profile around the area of the cut-off insulator core where surface blackening was clearly visible. The measurement was taken from the side of the biggest intensity of the coating towards the area of a lighter - grey - colour, towards the shed edge. The arched bend of the profile results from the shape of the shed

TABLE 2

The upper surface of the $P f$ insulator middle shed sample - the surface profile parameters within the area of a variable layer of the dark coating

\begin{tabular}{|c|c|c|c|c|c|}
\hline \hline Parameter & Ra & Rq & Rp & Rv & $\mathbf{R z}$ \\
\hline Value $[\mu \mathrm{m}]$ & 2.5 & 3.1 & 8.8 & 6.0 & 14.8 \\
\hline
\end{tabular}


On the lower surface of the middle shed sample, the measurement was taken within the area around the cut-off insulator core. It contained a distinct light path, clearly visible in the image of the profile - Figure 7. The profile parameters, strongly elevated with respect to the original ones (Table 1), were presented in Table 3.
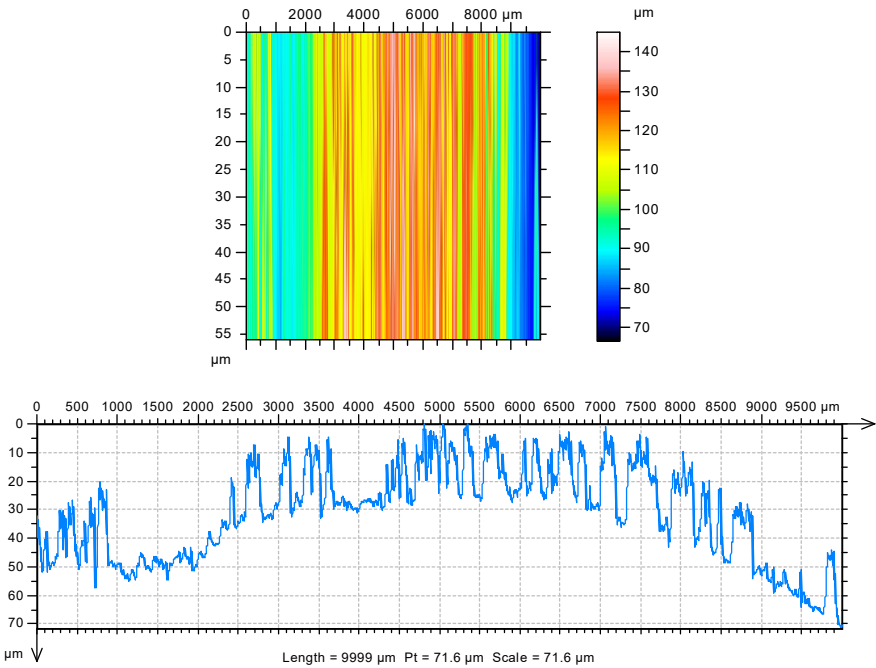

Fig. 7. The lower surface of the $P f$ insulator middle shed sample - the image of the surface profile with a clear light path. Above - the image of the fivefold profile testing at a distance of $8 \mu \mathrm{m}$. The arched bend of the profile results from the shape of the shed

TABLE 3

The lower surface of the $P f$ insulator middle shed sample - the profile parameters of the surface of the strongly blackened area in the vicinity of the cut-off insulator core, with a clear light path

\begin{tabular}{|c|c|c|c|c|c|}
\hline \hline Parameter & Ra & $\mathbf{R q}$ & $\mathbf{R p}$ & $\mathbf{R v}$ & $\mathbf{R z}$ \\
\hline Value $[\mu \mathrm{m}]$ & 5.7 & 6.7 & 14.9 & 12.4 & 27.3 \\
\hline
\end{tabular}

It should be emphasized that irrespective of the dark coating reported on all $P f$, samples following the circular test, and the frequently observed light erosive paths, the most important damage was the cross puncture through the thickness of the shed. The punctures, as observed on the two samples under examination, result in the negative outcome of the circular test performed on the $P f$ insulators - Figures 4,5 and 8 .

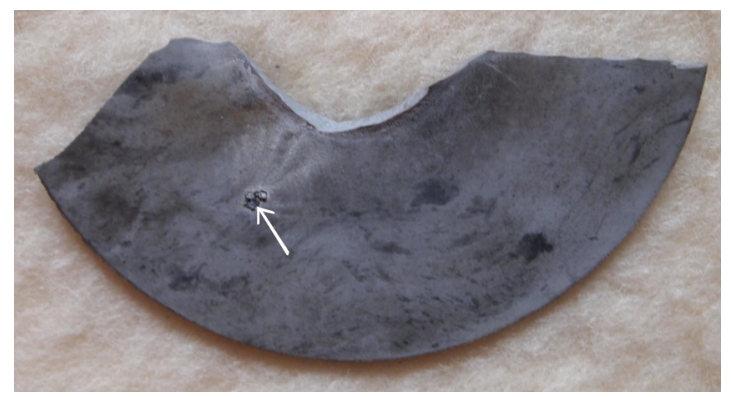

Fig. 8. The lower surface of the $P f$ insulator middle shed sample with an arrow pointing at the location of the puncture

\section{Examination of surface topography of $L t$ samples after the wheel test}

Like in the case of the $P f$ samples, the examination of the surface parameters of the sheds of $L t$ insulator, after the circular test, were performed on 6 samples. The upper and lower surfaces of the samples were tested, taken from the top, middle and bottom sheds of two insulators.

The $L t$ insulators shed samples after the wheel test had a dark coating on the surface. Its thickness and distribution varied, as in the case of the $P f$ samples. The coating was, however, thinner and had a smoother surface, the same as the rubber itself. It could have been removed easily, uncovering the surface - parameters of which were typical for the original samples.

The increase in the surface parameters at the location of the coating was clearly lower than for the $P f$ samples. Still, in case of a stronger blackening of areas neighbouring the cut-off insulator core, significant changes were recorded regarding the image and parameters of the surface profiles. Even small cracks on a layer of black sediment also had a significant impact on the increase in the profile parameters. It should be emphasized that no sample featured the effect of a cross puncture.

As exemplary and representative, resulting from a great number of tests made on $L t$ samples, the test was presented of the middle shed sample of one of the two insulators under examination. It demonstrated clear effects of electric discharges, both on the upper and lower side. Those were, in particular, areas in the vicinity of the cut-off insulator core - Figures 9 and 10.

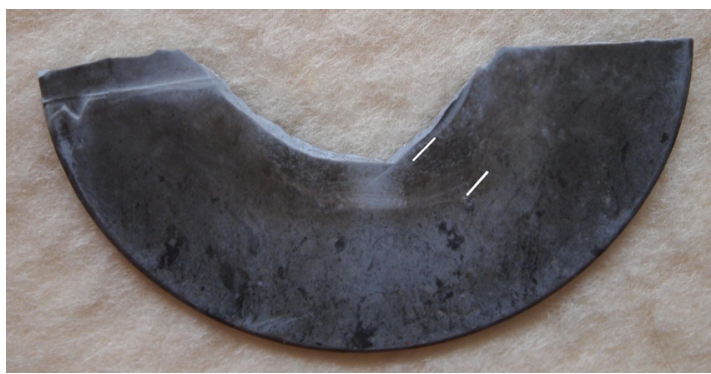

Fig. 9. The upper surface of the $L t$ insulator middle shed sample. The lines denote the area under examination with a bigger intensity of and small cracks in the dark coating. The measurement was taken towards the shed edge

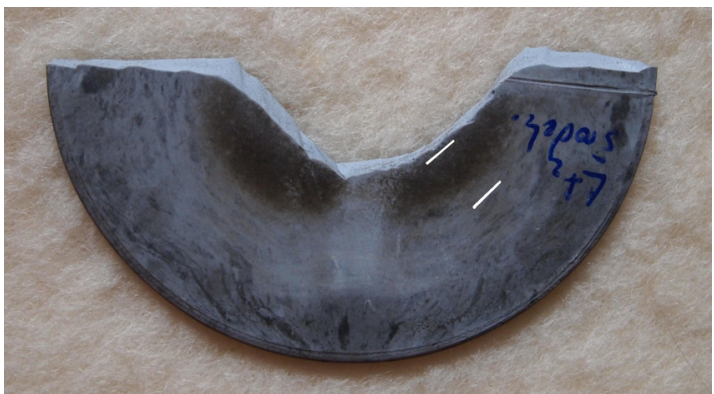

Fig. 10. The lower surface of the $L t$ insulator middle shed sample. Dark sediment covering is visible of the surface within the area around the cut-off insulator core. The measurement in the area marked with lines was taken towards the shed edge 
In addition, on the upper side of the sample, small cracks were observed in a layer of dark coating.

As expected, on both surfaces neighbouring the cut-off insulator core, changes were recorded in the surface profile image and parameters - Figures 11 and 12, Tables 4 and 5. A slightly higher increase in the profile parameters was found in the case of the upper surface (Table 4). Although the coating layer on the lower side seems darker and thicker, upon closer observation, on the upper side (Fig. 9), small cracks within the layer of black sediment were observed. Their presence probably resulted in a stronger increase of the surface parameters and the occurrence of bigger peaks on its profile - Figure 11 and Table 4 .
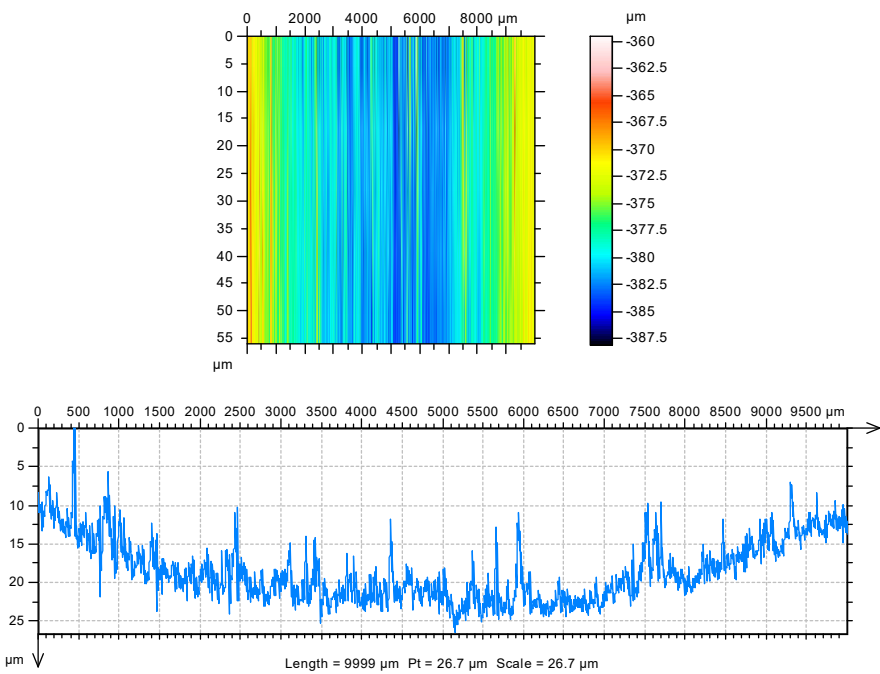

Fig. 11. The upper surface of the $L t$ insulator middle shed sample - the image of the surface profile of the blackened area in the vicinity of the cut-off insulator core. Above - the image of the fivefold profile testing at a distance of $8 \mu \mathrm{m}$. The arched bend of the profile results from the shape of the shed

TABLE 4

The upper surface of the $L t$ insulator middle shed sample - the parameters of the surface profile of the blackened area in the vicinity of the cut-off insulator core. The values are significantly elevated with respect to the original (reference) surfaces under examination

\begin{tabular}{|c|c|c|c|c|c|}
\hline \hline Parameter & Ra & $\mathbf{R q}$ & $\mathbf{R p}$ & $\mathbf{R v}$ & $\mathbf{R z}$ \\
\hline Value $[\mu \mathrm{m}]$ & 1.3 & 1.8 & 7.2 & 4.1 & 11.3 \\
\hline
\end{tabular}

TABLE 5

The lower surface of the $L t$ insulator middle shed sample the parameters of the surface profile of the strongly blackened area in the vicinity of the cut-off insulator core. The values are clearly elevated with respect to the original (reference) surfaces under examination

\begin{tabular}{|c|c|c|c|c|c|}
\hline Parameter & Ra & $\mathbf{R q}$ & $\mathbf{R p}$ & $\mathbf{R v}$ & $\mathbf{R z}$ \\
\hline Value $[\mu \mathrm{m}]$ & 1.2 & 1.6 & 5.5 & 4.0 & 9.5 \\
\hline
\end{tabular}

It should be emphasized that the effects of the wheel test were weaker in the case of the $L t$ insulator shed samples than for the $P f$ samples. First of all, no cross puncture occurred of any of the sheds of both insulators under examination. The
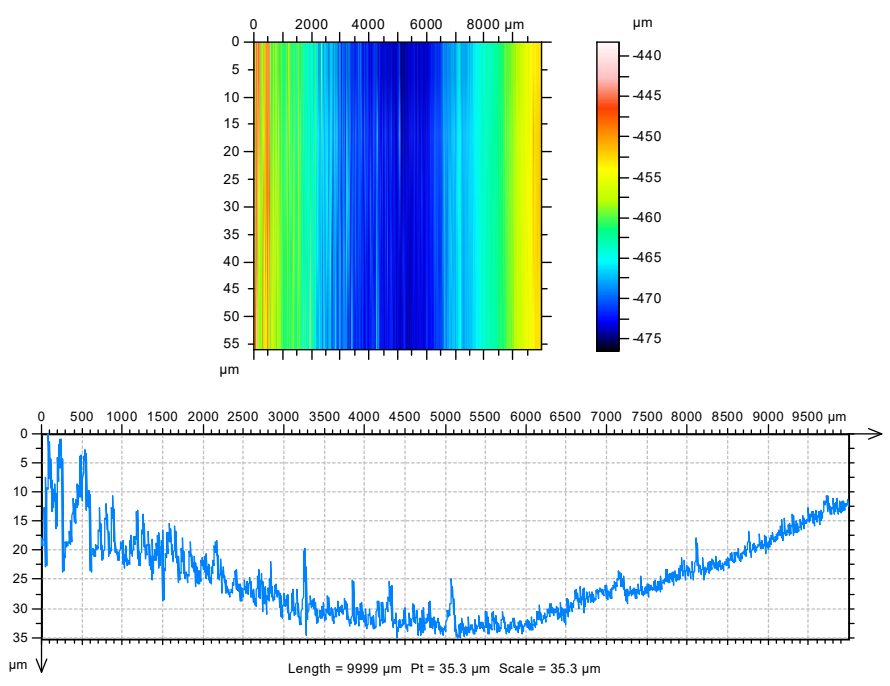

Fig. 12. The lower surface of the $L t$ insulator middle shed sample - the image of the surface profile within the area of increased intensity of black coating, in the vicinity of the cut-off insulator core. The measurement taken towards the shed edge - according to the decreasing thickness of the layer. The last part of the graph corresponds to a very weakly soiled surface. Above - the image of the fivefold profile testing at a distance of $8 \mu \mathrm{m}$. The arched bend of the profile results from the shape of the shed

increase of the surface parameters at the place where the sediment occurred was much smaller. The resulting sediment had surface parameters more similar to the original material, which was glossy and smooth to the touch, as opposed to the matt and rough $P f$ material.

\section{Examination by means of micro indentation method}

The scope of the testing of the samples by means of the micro indentation method was limited and mostly of comparative nature. These aimed to verify whether and to what extent both tested materials $-P f$ and $L t$ - are different in terms of compliancestiffness, and whether the action of electric discharges during the wheel test results in a change of the original characteristics of both elastomers.

The initial testing was performed in three randomly chosen places on the upper surface of the $P f$ insulator top shed sample, and in three randomly chosen places on the upper surface of the $L t$ insulator bottom shed sample. The force-penetration depth characteristics $(\mathrm{P}-\mathrm{h})$, as compared, are presented in Figure 13 .

Based on the measurements, as taken, of the original (reference) samples, good parameter recurrence was reported for the $L t$ material and slightly worse for the $P f$ material. It should also be emphasized that both elastomers demonstrate surprisingly big differences between each other. The $L t$ material is approximately three times stiffer - thus less compliant than the $P f$ material

In case of the $P f$ material, the measurement of micro indentation on the shed following the wheel test was taken in three places on the upper surface of the top shed. Two tests 


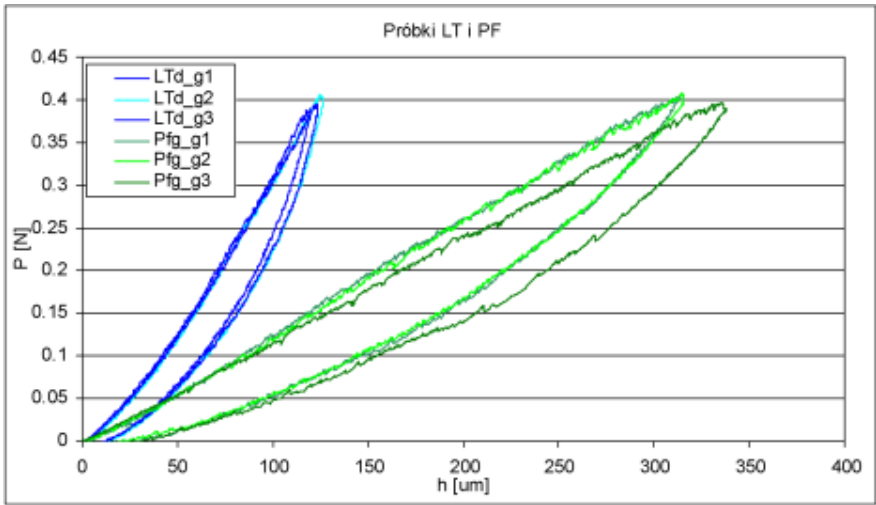

Fig. 13. The force-penetration depth characteristics compared. The measurements were taken in three places on the upper surface of the Pf insulator top shed sample - the green graphs, and in three places on the upper surface of the $L t$ insulator bottom shed sample - the blue characteristics

were performed within the area of strong blackening, whereas one measurement was taken next to it, yet on a surface that was almost void of the blackening. After the wheel test - of resistance to the effects of electric discharges - probably mainly due to the effect of temperature, there was a decrease in stiffness, hence the compliance of the $P f$ material increased. The shape of the graphs also underwent some change - becoming more bent, crescentic. The P-h characteristics of the three samples following the circular test demonstrated considerable differences from one another as well. The closest to the original - reference - curves was the characteristic of the test performed in the area almost void of the blackening. However, the results of the two measurements, as taken at a distance of $0.5 \mathrm{~cm}$ within the same small area of strong blackening, also demonstrated clear differences. This suggests surprisingly large variation of the stiffness-compliance of the $P f$ material as a result of the wheel test - Figure 14 .

In case of the $L t$ material, the measurement of micro indentation on the shed following the wheel test was taken in two places on the upper surface of the bottom shed. The first test was performed within the area of strong focused blackening, the other one - on a very weakly blackened surface. The shape of the characteristics obtained was, however, the same. Like for the $P f$ material, as a result of the wheel test, the stiffness was reduced, hence the compliance of the $L t$ material increased. The effect was a strong one and the change amounted to ca. $50 \%$. The shape of the graphs, too, underwent some change - becoming slightly less bent. The P-h characteristics of both $L t$ samples after the wheel test demonstrate, as opposed to the Pf material, very good recurrence. Despite the measurements being taken at points of a big difference in the thickness of the sediment.

Figure 14 presents the P-h characteristics, as compared, of the $P f$ and $L t$ material samples, following the wheel test of resistance to the effect of electric discharges. One can clearly see the much higher stiffness - lower compliance, as well as the much better recurrence of the characteristics for the $L t$ material.

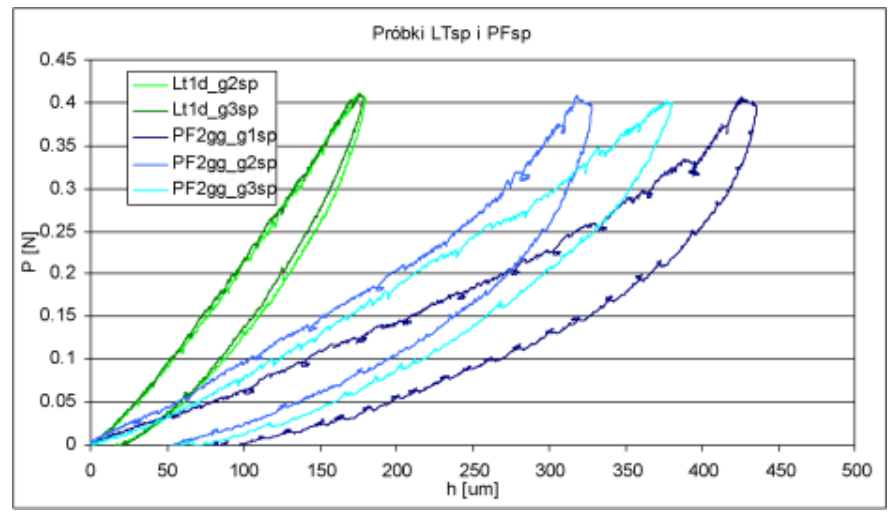

Fig. 14. The force-penetration depth characteristics, as compared, for the $P f$ sample measurements - the blue curves, and the $L t$ samples the green curves after the wheel test. The differences are noticeable regarding the stiffness (compliance) of both materials and the recurrence of results

\section{Conclusions}

The HTV (HCR) silicone rubbers under examination Pf and $L t$, used as housing material of composite insulators, despite their similar chemical composition, demonstrated considerable differences regarding the physical properties. The surface of the $P f$ shed samples was matt and slightly rough to the touch. On the other hand, the surface of the $L t$ shed samples was smooth and glossy. Sheds made of the rubber designed as $L t$ were also visibly thicker and perceptibly stiffer than in the case of the $P f$ sheds. This indicates differences regarding the manufacturing processes as applied by both manufacturers.

The testing of the surface topography (profile images and their parameters), as well as the force-penetration depth characteristics (P-h), performed by means of the micro indentation method, demonstrated clear differences too. They concerned both the original appearance of both materials and the degree of defecting of the surface as a result of the action of electric discharges during the wheel test.

The examination of the surface profile demonstrated satisfactory recurrence of the original parameters of the surface of both materials. With similar unevenness parameters, significantly higher values of the statistical parameters were reported, however, for the matt and rough $P f$ samples. The surfaces of all shed samples after the wheel test showed the occurrence of dark coating, which can yet be easily removed. Still, its thickness and distribution varied a lot with respect to specific samples. In general, more black sediment occurred on the surface of the $P f$ insulator samples. Regarding the $P f$ samples, a strong effect of the dark soiling layer on the surface profile parameters was reported. The unevenness amplitude increased significantly, as well as - in proportion to the thickness of the layer - the recorded surface parameters. Light erosive paths were also observed on some samples. These usually constituted valleys of several up to ca. $20 \mu \mathrm{m}$.

The most important damage, observed only on the $P f$ samples, was a cross puncture through the shed. This means that in 
the case of these insulators the result of the wheel test must be regarded as negative.

In case of the $L t$ shed samples, the wheel test caused significantly weaker effects. No cross puncture occurred of any of the sheds of both insulators under examination. The sediment layers were thinner and the increase in the surface parameters, as recorded was much smaller. The parameters of the blackened area were also closer to the original - smooth and glossy - material.

The measurement of the micro indentation of the original samples demonstrated good parameter recurrence for both materials, the $L t$ in particular. Both materials show big differences regarding the force-penetration depth characteristics $(\mathrm{P}-\mathrm{h})$ between each other. The $L t$ material is approximately three times stiffer - thus less compliant than the $P f$ material. As a result of the wheel test, probably mainly due to the effect of temperature, there was a significant decrease in stiffness, hence the compliance of both silicone materials - Pf and $L t$ - increased. The shape of the force-penetration depth characteristics $(\mathrm{P}-\mathrm{h})$ also underwent some changes.

The P-h characteristics of the $P f$ samples after the wheel test demonstrated surprisingly big differences from one another. On the other hand, the characteristics of the $L t$ samples demonstrated very good recurrence. Despite the measurements being taken at points of a big difference in the thickness of the sediment. In general, the significantly higher stiffness (lower compliance) and the much better recurrence of the characteristics for the $L t$ material should be emphasized.

The insulators of both types had passed the salt fog test resistance of the shed material to the effect of electric discharges - without any reservations. On the other hand, the result of the wheel test cannot be regarded as positive in the case of the $P f$ insulators. First of all, cross punctures occurred in several places. Besides, the sheds became covered with a dark coating of various thickness, and there were traces left by surface discharges. This demonstrates that the $P f$ material is less resistant to tracking and erosion, in relations to the $L t$ material, which can be surprising. It should be noted that the chemical composition of both HTV (HCR) silicone was very similar to each other but results of tests can be astonished. It seems that some difference in the thickness of sheds under testing cannot stand for only explanation of the obtained results. Clearly a quality of the applied rubbers and manufacturing process had to contribute to the results of the tests.

The results of the tests show also that effects of the aging factors are much stronger during the wheel test than in the case of the salt fog test. This may mean that these tests are hardly compatible, and the wheel test provided a better distinction regarding the physical properties of the materials under examination. It seems, therefore, that reintroduction of this test into standards, is justified.

\section{REFERENCES}

[1] IEC /TR $62730 \mathrm{HV}$ polymeric insulators for indoor and outdoor use tracking and erosion testing by wheel test and $5000 \mathrm{~h}$ test. Edition 1.0 2012-03.

[2] A.S. Krzma, M. Albano, R.T. Waters and A. Haddad, Comparative performance of $11 \mathrm{kV}$ silicone rubber polymeric insulators with HVAC and HVDC excitations using the rotating wheel dip test, paper no. 653, Mat. $19^{\text {th }}$ Int. Symp. on HV Engineering, Pilsen, Czech Republic, August 23-28, 2015.

[3] Standard IEC 62217:2012-09. Edition 2.0: Polymeric HV insulators for indoor and outdoor use-General definitions, test methods and acceptance criteria.

[4] M. Mackiewicz, Evaluation of the methods of examination of the resistance of insulator sheath and sheds to creep discharges and erosion. Report of the Institute of Power Engineering No. EWN/91/Stat/13 MMM, Part 1: Tests of the resistance of the composite insulators in the salt mist chamber and in the circular test, Warsaw, November 2013 (in Polish).

[5] Standard ISO 4287:1997: Geometrical Product Specifications (GPS) - Surface texture: Profile method - Terms, definitions and surface texture parameters.

[6] S. Kucharski, Z. Mróz, Identification of yield stress and plastic hardening parameters from a spherical indentation test, Int. J. Mech. Sci. 49, 1238-1250 (2007). 